\title{
Self-assembly mechanisms of short atomic chains on single-layer graphene and boron nitride
}

\author{
V. Ongun Özçelik ${ }^{1,2}$ and S. Ciraci ${ }^{1,2,3, *}$ \\ ${ }^{1}$ UNAM-National Nanotechnology Research Center, Bilkent University, Ankara 06800, Turkey \\ ${ }^{2}$ Institute of Materials Science and Nanotechnology, Bilkent University, Ankara 06800, Turkey \\ ${ }^{3}$ Department of Physics, Bilkent University, Ankara 06800, Turkey
}

(Received 3 July 2012; revised manuscript received 18 September 2012; published 12 October 2012)

\begin{abstract}
Nucleation and growth mechanisms of short chains of carbon atoms on single-layer, hexagonal boron nitride (h-BN) and short $\mathrm{BN}$ chains on graphene are investigated using first-principles plane-wave calculations. Our analysis starts with the adsorption of a single carbon adatom and examines its migrations. Once $\mathrm{a}_{2}$ nucleates on h-BN, the insertion of each additional carbon at its close proximity causes a short segment of carbon atomic chain to grow by one atom at at a time in a quaint way: The existing chain leaves its initial position and subsequently is attached from its bottom end to the top of the carbon adatom. The electronic, magnetic, and structural properties of these chains vertically adsorbed to h-BN depend on the number of carbon atoms in the chain, such that they exhibit an even-odd disparity. An individual carbon chain can also modify the electronic structure with localized states in the wide band gap of h-BN. As a reverse situation, we examined the growth of short BN atomic chains on graphene, which attribute diverse properties depending on whether $\mathrm{B}$ or $\mathrm{N}$ is the atom bound to the substrate. These results together with ab initio molecular dynamics simulations of the growth process reveal the interesting self-assembly behavior of the grown chains. Furthermore, we find that these atomic chains enhance the chemical activity of h-BN and graphene sheets by creating active sites for the bonding of various adatoms and can act as pillars between two and multiple sheets of these honeycomb structures, leaving wider spacing between them to achieve high-capacity storage of specific molecules.
\end{abstract}

DOI: 10.1103/PhysRevB.86.155421

PACS number(s): 68.65.-k, 73.22.-f, 75.75.-c, 81.07.-b

\section{INTRODUCTION}

After the synthesis of graphene, ${ }^{1,2}$ a monolayer of $s p^{2}$ bonded honeycomb structure of carbon atoms, interest has focused on two-dimensional nanostructures having honeycomb structures. Planar hexagonal boron nitride (h-BN) is an ionic honeycomb structure consisting of alternatively bonded boron and nitrogen atoms and is an analog of graphene. ${ }^{3,4}$ Despite the structural similarity, h-BN differs from graphene with its wide band gap and dielectric properties. ${ }^{5}$ Various boron nitride structures such as nanosheets, ${ }^{6}$ nanotubes, ${ }^{7}$ and nanowires ${ }^{8}$ have already been synthesized. Also, recent studies show that h-BN can be used to improve the current voltage properties of graphene transistors by improving the mobility of electrons in graphene as compared to graphene films on silicon substrates. ${ }^{9}$ These properties hold promise for novel technological applications of h-BN structures.

A thorough understanding of the properties of h-BN structure and investigating its functionalization is important for future applications of this wide-band-gap material. Carbon, being in the same row of the periodic table with boron and nitrogen, is one of those foreign atoms which can greatly change the physical and chemical properties of h-BN. In a recent study, ${ }^{10}$ the effects of adatoms adsorbed on $\mathrm{BN}$ were investigated using first-principles calculations, and it was shown that high coverage of carbon adatoms can change the magnetic properties and band gap of the system, whereas adatoms induce localized states in the band gap at low coverage.

Single carbon adatom and monoatomic chains of carbon atom are interesting entities, which can functionalize h-BN. A short segment of carbon atomic chain containing $n \mathrm{C}$ atoms, indicated as $\operatorname{CAC}(n)$ hereafter, is a truly one-dimensional carbon allotrope, and has recently drawn attention due to its linear geometry, high strength, size-dependent quantum ballistic conductance, and interesting electronic properties. These properties of CACs were both theoretically ${ }^{11-20}$ and experimentally ${ }^{21-23}$ investigated. Theoretical studies revealed the Peierls distortion in CACs, ${ }^{11,13,19}$ half-metallic and spintronic properties, ${ }^{16-18}$ size-dependent quantum conductance, ${ }^{12,14}$ and other geometrical structures. ${ }^{13}$ Chain structures of other group-IV elements and group III-V compounds were also treated. ${ }^{15}$ Concomitantly, carbon atomic chains are synthesized. ${ }^{21-23}$ Recently, it was also shown that CACs can be grown on graphene and modify its properties ${ }^{24}$ which have also been justified by the images obtained earlier using high-resolution transmission electron microscopy (TEM). ${ }^{25}$

In this paper, we studied the growth of short carbon and BN chains on single-layer h-BN and graphene, respectively. We first examined the adsorption of a single carbon atom on $\mathrm{h}-\mathrm{BN}$ by calculating its energy landscape and diffusion barrier. This is followed by the investigation of the nucleation and growth processes of CACs on h-BN. We performed both conjugate gradient calculations and molecular dynamics simulations in order to determine the stabilities and bonding properties of these CACs and show how they can grow on the plane of $\mathrm{BN}$ as new carbon atoms are introduced by one atom at a time at the close proximity of an existing CAC. Once these two materials, namely, carbon atomic chains and monolayer h-BN, are combined, fundamentally interesting properties are attained for promising future applications. Interestingly, the properties of the grown structures depend on the number of carbon atoms in the chains, such that they exhibit an even/odd disparity. In addition, we showed that CACs grown on h-BN constitute chemically active sites for $\mathrm{Au}, \mathrm{Li}$, and $\mathrm{H}_{2}$, which are normally weakly bonded to h-BN. For example, 
$\mathrm{H}_{2}$ approaching to the top of the $\mathrm{CAC}(2)$ is dissociated and separated into two $\mathrm{H}$ atoms, each attached to the chain. We also showed that CACs can attach to another h-BN from its free end to serve as pillars to increase the spacing between $\mathrm{h}-\mathrm{BN}$ flakes, where molecules such as $\mathrm{H}_{2}$ can be stored. We presented the electronic-energy band structure for various lengths of chains to see their variations with the number of chain atoms. We finally studied the problem from the reverted point of view and presented the growth patterns, optimized structural and electronic structures of BN chains grown on graphene surface. Our results revealed the unique self-assembly character of carbon and $\mathrm{BN}$ chains on single-layer honeycomb structures and interesting features attained thereof.

\section{METHOD}

In our calculations, we use the state-of-the-art firstprinciples plane-wave calculations within the densityfunctional theory ${ }^{26,27}$ combined with $a b$ initio, finitetemperature molecular dynamics (MD) calculations using projector augmented wave potentials. ${ }^{28,29}$ The exchangecorrelation potential is approximated by the generalized gradient approximation with van der Waals correction. ${ }^{30,31}$ Numerical computations have been carried out by using VASP software. $^{32}$ A plane-wave basis set with energy cutoff of $600 \mathrm{eV}$ is used. The Brillouin zone is sampled in the $\mathbf{k}$ space within the Monkhorst-Pack scheme, ${ }^{33}$ and the convergence of the total energy and magnetic moments with respect to the number of $\mathbf{k}$ points is tested. The convergence for energy is chosen as $10^{-5} \mathrm{eV}$ between two consecutive steps. In the relaxation of structures and band-structure calculations, the smearing value for all structures is taken as $0.01 \mathrm{eV}$. We consider adsorption of chains on $(4 \times 4)$ supercells and treat the system using periodic boundary conditions. The pressure on each system was kept smaller than $\sim 2 \mathrm{kBar}$ per unit cell in the calculations. In the ab initio MD calculations, the time step was taken as $2.5 \mathrm{fs}$ and atomic velocities were renormalized to the temperature set at $T=500$ and $1000 \mathrm{~K}$ at every 40 time steps. In the MD stability tests, the simulations were run for $10 \mathrm{ps}$.

\section{ADSORPTION OF SINGLE CARBON ADATOM ON H-BN}

Before going into detailed studies of carbon chains, we first investigate the adsorption and migration of the single carbon adatom, which is the starting point of CAC growth on the h-BN substrate. We use a $(4 \times 4)$ supercell of h-BN that consists of 16 boron and 16 nitrogen atoms. There is an energy difference of $\sim 0.2 \mathrm{eV}$ between the spin-polarized and spin-unpolarized energy values in favor of the spin-polarized state, indicating that the system has a magnetic ground state. Therefore, all of the calculations mentioned hereafter are performed using the spin-polarized conditions.

The most favorable binding site of the single carbon atom was determined by placing the adatom initially to various adsorption sites at a height of $\sim 2 \AA$ from the $\mathrm{BN}$ atomic plane and running fully self-consistent geometry optimization calculations by keeping the adatom fixed in the $x$ and $y$ directions and letting the vertical $z$ coordinate of the adatom,
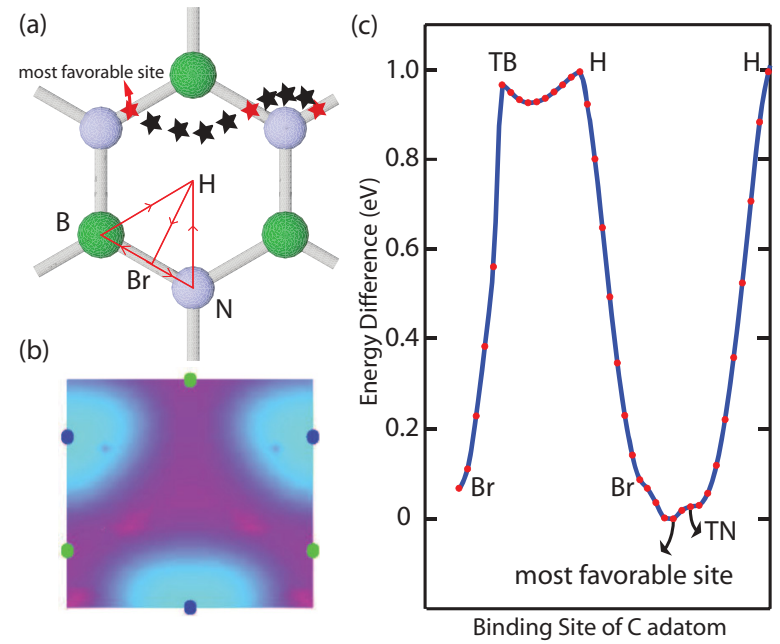

FIG. 1. (Color online) Energy variation of single carbon atom adsorbed on various sites of single-layer 2D hexagonal boron nitride structure $(\mathrm{h}-\mathrm{BN})$ calculated in a $(4 \times 4)$ supercell. (a) h-BN honeycomb structure on which the adsorption energies are calculated. Nitrogen and boron atoms are represented by blue and green balls, respectively. The most favorable binding site of carbon adatom is marked by the red star in the figure. The path of diffusion of carbon adatom with the minimum energy barrier of $\sim 0.65 \mathrm{eV}$ is indicated by stars. (b) Complete energy landscape of $\mathrm{C}$ adatom on h-BN structure. Light blue regions show favorable sites and the energy barrier further increases as the color goes to dark blue and purple. (c) Energy variation of carbon adatom is shown along the path indicated by red arrows in (a). The energy difference between the most favorable site (indicated by red star) and the bridge (Br), top of boron (TB), hollow $(\mathrm{H})$, top of nitrogen $(\mathrm{TN})$ sites are calculated as $0.07,0.95$, 1.00 , and $0.03 \mathrm{eV}$, respectively.

which is its height from the plane, free. Meanwhile, the atoms in the BN supercell are relaxed in all directions except for one corner atom of the supercell, which is fixed in all directions to prevent h-BN from sliding.

In Fig. 1(a), the nitrogen and boron atoms of the optimized h-BN structure are separated from each other by $1.45 \AA$. The most favorable bonding site of the single carbon adatom, which turns out to be near the top site of the nitrogen atom, is marked with a red star. The migration (diffusion) path of the carbon adatom on h-BN with a minimum energy barrier is shown by black stars. The minimum energy barrier is calculated as $0.68 \mathrm{eV}^{34}$ The energy landscape calculated over the whole $\mathrm{BN}$ hexagon also shows that the energy barrier to the diffusion further increases as the carbon atom moves away from the nitrogen atom as shown in Fig. 1(b). The variation of energy calculated along the two-dimensional (2D) path shown in Fig. 1(a) is presented in Fig. 1(c). As indicated in the figure, the most favorable site for the carbon atom is near the top nitrogen site, although not exactly on top of nitrogen. The energy barrier between the most favorable site and the top bridge (BR), top boron (TB), hollow (H), and top nitrogen (TN) sites were calculated as $0.07,0.95,1.00$, and $0.03 \mathrm{eV}$, respectively.

In addition to the diffusion path analysis of a single carbon adatom, we next study the interaction between two carbon atoms on the h-BN surface. When the distance between the two adatoms becomes less than a threshold distance of $\sim 2 \AA$, 


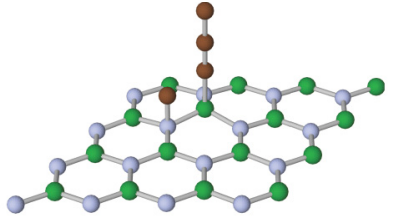

(i)

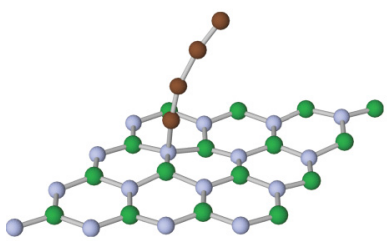

(iii)

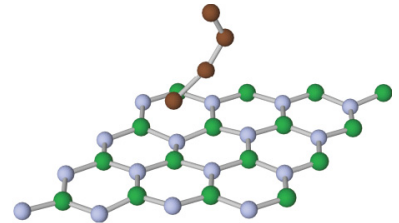

(ii)

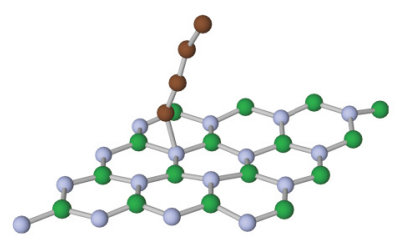

(iv)
FIG. 2. (Color online) Snapshots of the molecular dynamics simulation showing the formation of a short chain comprising four carbon atoms. The snapshots correspond to the initial, 20th, 40th, and 120th steps of the molecular dynamics simulation done at $500 \mathrm{~K}$. Note that the formation of CAC(4) takes place as the CAC(3) leaves its initial bonding position and attaches to a single carbon adatom at close proximity from its top. Similar growth mechanism is also seen during the formation of CACs of length $n \leqslant 8$.

these two carbon atoms attract each other and form a CAC(2) perpendicularly attached to $\mathrm{h}-\mathrm{BN}$. This is indeed the nucleation for longer CACs. The most favorable binding site $\mathrm{CAC}(2)$ is again near the top site of nitrogen. A complete site analysis was also performed to confirm this result by placing a CAC(2) on various adsorption sites and comparing the total energy values.

\section{GROWTH OF CARBON ATOMIC CHAINS ON H-BN}

\section{A. Chain growth and even/odd disparity}

Growth of the CAC further continues when a third carbon adatom is introduced at the close proximity of $\mathrm{CAC}(2)$. However, this time a complete site analysis of $\mathrm{CAC}(3)$ shows that the most favorable bonding site is the top of the boron atom, instead of the nitrogen site. The formation of CAC(3) happens as follows: $\mathrm{CAC}(2)$ leaves its initial bonding position, moves higher from the $\mathrm{BN}$ plane, and in the mean time it gets closer to the single adatom until they are bound to each other near the new energetic site, which is the top of the boron site. Similar chain growth behavior is also seen during formation of CACs at different lengths. This process is further investigated with $a b$ initio molecular dynamics simulations at $500 \mathrm{~K}$ and the snapshots taken from the growth of CAC(4) is presented in Fig. 2. We initiate the MD simulation by placing a carbon adatom and a $\mathrm{CAC}(3)$ to their bonding sites as shown in Fig. 2(i). The simulation was run for 2000 time steps and snapshots taken from the initial, 20th, 40th, and 120th time steps are shown. As the simulation proceeds to the 2000th step, the chain stays in its position shown Fig. 2(iv), which is an indication of its stability at that bonding site.

We further continue the above analysis with fourth, fifth, sixth, and seventh carbon adatoms. In general, once a new carbon atom is implemented at the close proximity of the

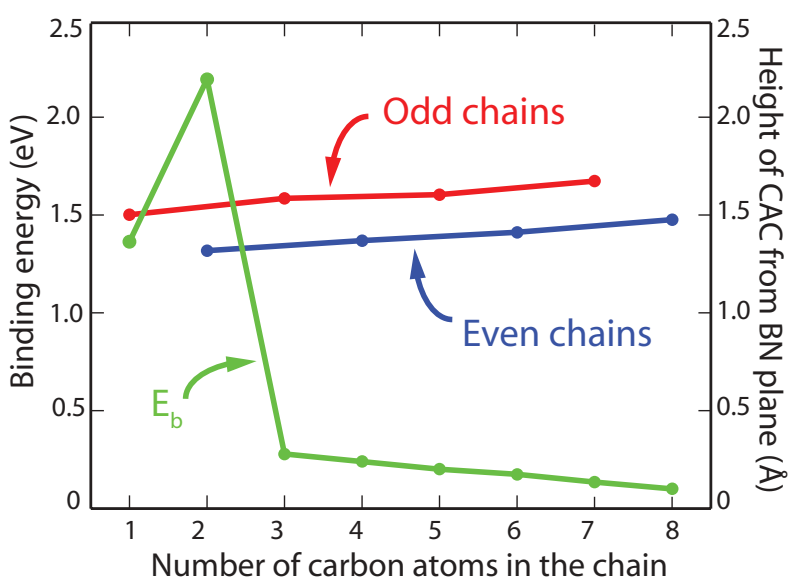

FIG. 3. (Color online) Binding energies $\left(E_{b}\right)$ and the heights $(h)$ of odd- and even-numbered $\mathrm{CAC}(n)$ 's from the atomic plane of $\mathrm{BN}$ are shown in green, red, and blue lines, respectively. The $h$ values exhibit an even/odd family behavior depending on the number of carbon atoms in the chain. The sudden peak in the binding energy arises from the change of the magnetic state of $\mathrm{CAC}(2)$ from magnetic to nonmagnetic when it binds to hexagonal $\mathrm{BN}$.

existing chain $\mathrm{CAC}(n-1)$, this chain leaves its binding site and is bound to the top of a new carbon adatom in its new most favorable binding site. Namely, this binding site keeps changing between the near-top nitrogen site and the near-top boron site. With the exception of the single carbon adatom, we observe an even/odd disparity of the binding site depending on the number of atoms in the $\mathrm{CAC}$, that is, even-numbered CACs bind to the h-BN substrate near the top nitrogen site, and the odd-numbered CACs bind near the top boron site. This situation can be attributed to the charge density of $\operatorname{CAC}(n)$ at its end, which depends whether $n$ is even or odd.

The binding energies of CACs attached to graphene are calculated using the expression $E_{b}=E_{T}$ [free-linear $\mathrm{CAC}(n)]+E_{T}\left[\right.$ bare h-BN]- $E_{T}[\mathrm{CAC}(n)+\mathrm{h}-\mathrm{BN}]$. In this expression, $E_{T}$ [free-linear CAC $\left.(n)\right]$ is the total energy of fully relaxed linear chain of length $n, E_{T}$ [bare h-BN] is the total energy of bare $(4 \times 4)$ supercell of h-BN, and $E_{T}[\mathrm{CAC}(n)+$ h$\mathrm{BN}]$ is total energy obtained when $\mathrm{CAC}(n)$ binds to the supercell of h-BN. The complete list of these binding energies and the binding geometries of these CACs are presented in Table I.

It is seen that, with the exceptional case of $\mathrm{CAC}(2)$, which is further discussed in the following section, the binding energies $\left(E_{b}\right)$ of the CACs tend to decrease as the length of the chains (or the number of carbon atoms) increase. Similarly, due to the decrease in the binding energy, the height $(h)$, namely, the distance between the h-BN plane and the first carbon atom of the CAC, increases. However, the $h$ values also show an even/odd disparity. In other words, there is an increasing trend in the $h$ values when the CACs are grouped as even- and odd-numbered chains, but the height of $\mathrm{CAC}(2 n)$ is always less than $\mathrm{CAC}(2 n-1)$, as shown in Fig. 3. This situation can be explained by the fact that the $\mathrm{C}-\mathrm{N}$ bond length is shorter than the C-B bond length, and $\mathrm{CAC}(2 n)$ is a more stable structure since it has a more symmetrical charge distribution 
TABLE I. Most favorable binding sites, total binding energies $\left(E_{b}\right)$, magnetic moments $(\mu)$, heights $(h)$ of $\mathrm{CAC}(n)$ from the $\mathrm{BN}$ plane, and the distances of the lowest carbon atom of the chain from the nitrogen $\left(d_{\mathrm{C}-\mathrm{N}}\right)$ and the boron $\left(d_{\mathrm{C}-\mathrm{B}}\right)$ atom in the BN plane for different $n$ values of carbon chains. The bonding sites and magnetic properties of CACs on BN exhibit an even/odd disparity. With the exception of the single carbon adatom, even-numbered CACs bind to BN near the top of the nitrogen (TN) atom and the odd-numbered CACs bind near the top of the boron (TB) atom. Additionally, the even- and odd-numbered chains grown on BN have magnetic and nonmagnetic (NM) ground states, respectively, with the exception of $\mathrm{CAC}(1)$ and $\mathrm{CAC}(2)$ cases. All calculations were performed on a $4 \times 4$ supercell which contains 32 carbon atoms.

\begin{tabular}{|c|c|c|c|c|c|c|}
\hline Structure & Site & $E_{b}(\mathrm{eV})$ & $\mu\left(\mu_{B}\right)$ & $h(\AA)$ & $d_{\mathrm{C}-\mathrm{N}}(\AA)$ & $d_{\text {C-B }}(\AA)$ \\
\hline $\mathrm{BN}+\mathrm{C}$ & $\sim \mathrm{TN}$ & 1.36 & 2.00 & 1.50 & 1.59 & 1.73 \\
\hline $\mathrm{BN}+\mathrm{CAC}(2)$ & $\sim \mathrm{TN}$ & 2.19 & NM & 1.32 & 1.46 & 1.83 \\
\hline $\mathrm{BN}+\mathrm{CAC}(3)$ & $\sim \mathrm{TB}$ & 0.28 & NM & 1.59 & 2.16 & 1.62 \\
\hline $\mathrm{BN}+\mathrm{CAC}(4)$ & $\sim \mathrm{TN}$ & 0.24 & 2.00 & 1.37 & 1.50 & 1.63 \\
\hline $\mathrm{BN}+\mathrm{CAC}(5)$ & $\sim \mathrm{TB}$ & 0.20 & $\mathrm{NM}$ & 1.60 & 2.28 & 1.62 \\
\hline $\mathrm{BN}+\mathrm{CAC}(6)$ & $\sim \mathrm{TN}$ & 0.18 & 1.97 & 1.41 & 1.52 & 1.58 \\
\hline $\mathrm{BN}+\mathrm{CAC}(7)$ & $\sim \mathrm{TB}$ & 0.14 & NM & 1.67 & 2.41 & 1.64 \\
\hline $\mathrm{BN}+\mathrm{CAC}(8)$ & $\sim \mathrm{TN}$ & 0.08 & 1.96 & 1.48 & 1.56 & 1.59 \\
\hline
\end{tabular}

as compared to $\mathrm{CAC}(2 n-1)$. These decrease $h$ distances of the even-numbered $\mathrm{CACs}$ that are bound to the nitrogen atom.

We finally test the stabilities of these h-BN $+\mathrm{CAC}(n)$ complexes at finite temperatures with molecular dynamics simulations. The $a b$ initio MD calculations were carried out for $10 \mathrm{ps}$ at $T=500 \mathrm{~K}$. The final stable bonding configurations of various CACs are shown in Fig. 4. After MD simulations, these CACs on h-BN remain stable and they do not detach from the BN plane, but they slightly pull the nearest B and $\mathrm{N}$ atoms of h-BN upwards from the plane, as seen from the side views given in Fig. 4(a). Also, the chains are not perfectly perpendicular to the $\mathrm{BN}$ plane, but are slightly tilted at different angles. Figure 4(b) shows the top views of the CACs, where only the carbon atoms closest to the BN planes are shown. As noted above, the even/odd disparity in the bonding sites of the chains with the exception of the single carbon atom can be seen. It should be noted that $a b$ initio MD simulations carried out for $10 \mathrm{ps}$ is not sufficiently long to accumulate enough statistics. However, calculations at high temperatures are worthwhile to give a hint about the stability at relatively lower temperatures. An unstable chain would easily break down or detach from the substrate. This did not happen in this case. Additionally, calculations using the conjugate gradient method resulted in chain structures bound to the substrate with significant binding energies, especially for short CACs. Hence, these tests provide sufficient evidence that the chains we studied are stable and remain attached to the single-layer h-BN.

\section{B. Electronic and magnetic properties}

Having found the structural properties of CACs on h-BN, we next focus on the variations of magnetic and electronic properties of h-BN with various lengths of CACs attached. First, we perform both spin-polarized and spin-unpolarized energy minimization calculations for free (bare) CACs without attaching them to h-BN and compare the minimum energies of magnetic and nonmagnetic cases. In a similar manner with the optimized structures of CACs attached to h-BN, it turns out the magnetization of free CACs also depends on the number $n$ of carbon atoms, namely, that a free chain has magnetic ground state if it has even number of carbon atoms, and a nonmagnetic ground state if it has odd number of carbon atoms with the exception of a single carbon atom in vacuum. Such an even/odd disparity in the magnetic moments of finite-size CACs was previously reported. ${ }^{19}$ Hence, when these chains are adsorbed to the h-BN sheet, the overall magnetic properties of the $h$ $\mathrm{BN}+\mathrm{CAC}(n)$ structures are maintained with the exception of $\mathrm{CAC}(2)$. While all other CACs preserve their magnetic ground states when bonded to h-BN, CAC(2) flips from the magnetic ground state to the nonmagnetic ground state. This change in the magnetic moment of $\mathrm{CAC}(2)$ increases its bond strength with h-BN and causes a higher binding energy. This is the reason for the sudden jump in Fig. 3.

The disparities observed so far are closely related to the bond-length variation of CACs with even and odd number of atoms. ${ }^{19}$ First, we note that the final stable structures of short CACs grown on h-BN have nonuniform bond-length distribution. While the even-numbered chains have polyyne type of structure with alternating short and long bonds, the oddnumbered chains have symmetrical bond-length variations around their center points. In other words, the bond-length variations for the odd chains reach to zero at the center, whereas the variation of bond lengths continues in chains having even $n$. This situation is related to the geometrical linear symmetry of the chain as also observed in a recent study for bare chains. ${ }^{35}$ For the odd chains, the geometrical center of the chain is the middle carbon atom since there are an even number of bonds. On the other hand, even chains have odd number of bonds and the geometrical center is on the middle bond. Since these chains are bonded to the h-BN substrate only from one side, they are free to move in the other direction. Hence, the chains need to be symmetrical around the center, and symmetrically equivalent bonds must have the same length. As a result, the two equivalent bonds around the center atom of the odd chains have the same length. Variations of bond lengths of h-BN $+\mathrm{CAC}(n)$ for $n=5-8$ are presented in Fig. 5 .

This overall symmetry in the geometrical structure of the odd-numbered chains also leads to a symmetry in the distribution of the magnetic moments, making the ground states singlet for odd chains. In the singlet state, magnetic moments exerted by the electrons cancel each other, leading 
$\mathrm{CAC}(1)$ $\operatorname{CAC}(2)$ CAC(3) CAC(4) $\mathrm{CAC}(5)$ $\mathrm{CAC}(6)$

(a)
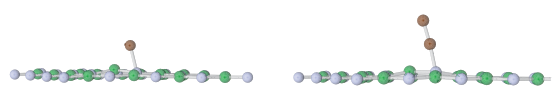

(b)
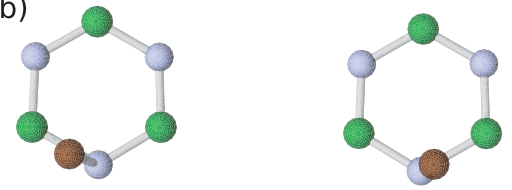
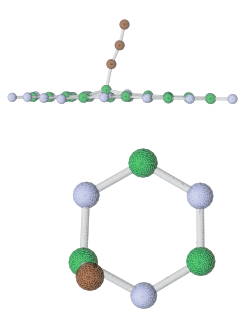
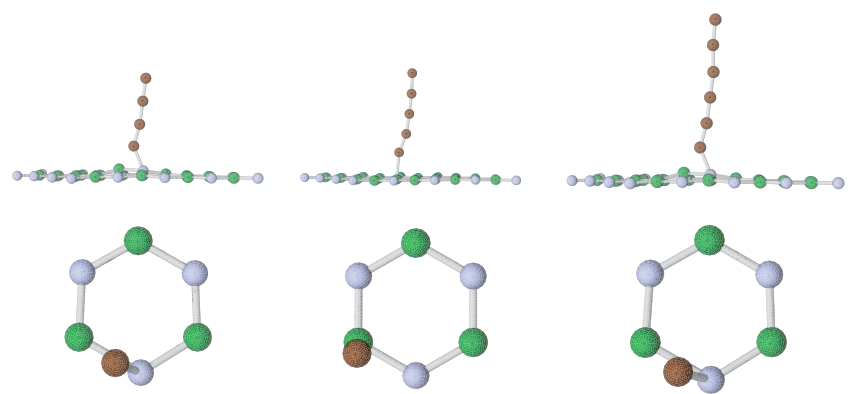

FIG. 4. (Color online) Side and top views of the most favorable binding configurations of CAC $(n)$ on hexagonal BN are shown in (a) and (b). N, B, and C atoms are represented by blue, green, and brown balls, respectively. CAC $(n)$ 's having an even number of carbon atoms (even $n$ ) are bound to $\mathrm{BN}$ near the top of nitrogen atom, whereas $\mathrm{CAC}(n)$ 's with odd number of carbon atoms (odd $n$ ) prefer the top boron site, with the exception of single carbon adatom. The geometries are calculated for a $(4 \times 4)$ supercell and their stabilities are also tested with MD simulations at $T=500 \mathrm{~K}$ for $10 \mathrm{ps}$. In (b), only the carbon atom of the chain that is closest to the BN plane is shown.

to zero net spin and zero magnetic moment. Therefore, these structures have a nonmagnetic ground state. On the other hand, even chains have an uneven distribution of magnetic moments on atoms leading them to have magnetic ground states. This magnetic preference also alters the binding sites of the chains accordingly. One would expect this situation to be reverted for chains saturated from both ends because the boundary conditions for the chains are altered as shown in a recent study. ${ }^{19}$

An individual $\mathrm{CAC}(n)$ attached to h-BN can modify the electronic band structure of h-BN. If an adsorbed $\mathrm{CAC}(n)$ is sufficiently far from others, it gives rise to localized states in the band gaps and resonance states in the band continua of h-BN. In this study, we consider a single $\mathrm{CAC}(n)$ adsorbed to h-BN using a model where one $\mathrm{CAC}(n)$ is attached to each repeating $(4 \times 4)$ supercell with the condition that interactions between adjacent CACs are negligible. This model recovers approximately the $(4 \times 4)$ folded bands of bare $h-B N$, except that the energy difference between the top of the valance bands and the bottom of the conduction bands gradually increases from 4.5 to $4.8 \mathrm{eV}$. Additionally, flat bands due to $\mathrm{CAC}(n)$ occurs in the band gap. These flat bands actually correspond to the localized states due to $\mathrm{CAC}(n)$. In Fig. 6(a),

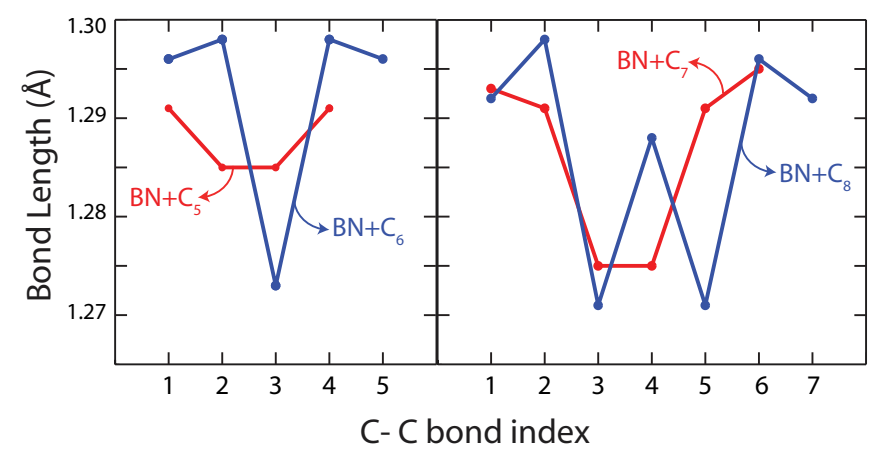

FIG. 5. (Color online) Variation of C-C bond lengths in the short carbon atomic chains $\mathrm{CAC}(n)$ grown on $\mathrm{BN}$. While the even-numbered chains (blue) have alternating short and long bonds, the odd chains (red) have a symmetrical bond length variation around their central atom. Note that the number of bonds in a chain is $n-1$. we present the electronic energy structure of h-BN $+\mathrm{CAC}(n)$ calculated for $n=1,2,3$, and 4 using supercell geometry explained above. The energy positions of the filled and empty flat bands in the gap are closely related with the energy levels of the corresponding $\operatorname{CAC}(n)$, which vary with $n$. For the magnetic $n=1$ case, spin-up states (i.e., flat bands) originating mainly from C- $p_{x y}$ orbitals occur above the top of the valence band. Empty spin-down states of the same orbital character occur near mid-gap. In the nonmagnetic case of $n=2$, spin-degenerate C- $p_{x y}$ and C- $p_{z}$ states are filled and occur near the top of the valence band. While a filled C- $p_{x y z}$ state touches the top of the valence band both for the nonmagnetic $n=3$ case and the magnetic $n=4$ case, the positions of empty C- $p_{x y}$ states in the band gap strongly depend on $n$. For all $n$ 's, the resonance states occur in the valence band within the energy of $2 \mathrm{eV}$ from the top.

The difference charge density is calculated by subtracting the charge densities of bare h-BN and bare $\mathrm{CAC}(n)$ from that $\mathrm{CAC}(n)+\mathrm{h}-\mathrm{BN}$ system by keeping the atomic configuration of adsorbed and bare CAC unaltered. In Fig. 6(b), the isosurfaces of difference charge density indicate that important changes from the corresponding bare CAC are found where $\mathrm{CAC}$ is bound to h-BN.

\section{Applications of CACs on BN}

Apart from its interesting and fundamental aspects, the growth of CACs on single-layer graphene is a reality and they can modify graphene's physical and chemical properties. Experimental observations and images obtained using highresolution TEM (Ref. 25) indicates that CACs can be formed on monolayer graphene surfaces. In this study, we show that $\mathrm{BN}$ is also a suitable substrate for the growth of $\mathrm{CAC}(n)$. Previous experimental studies ${ }^{4}$ and density-functional results ${ }^{36,37}$ have shown that epitaxial graphene grown on boron nitride surface is possible by means of the chemical vapor deposition technique or exposure of $\mathrm{BN}$ layer to carbon atoms or dimers at long time intervals. In these studies, it was noted that defected graphene structures with undesired chains can also form during the growth of graphene..$^{20,22}$ Thus, since the temperature range of our MD calculations is suitable 
(a)

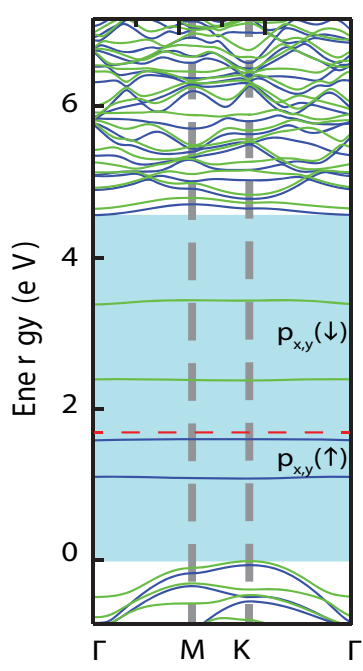

(b)

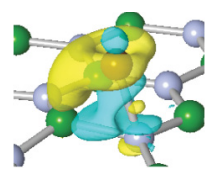

$\mathrm{BN}+\mathrm{CAC}(2)$
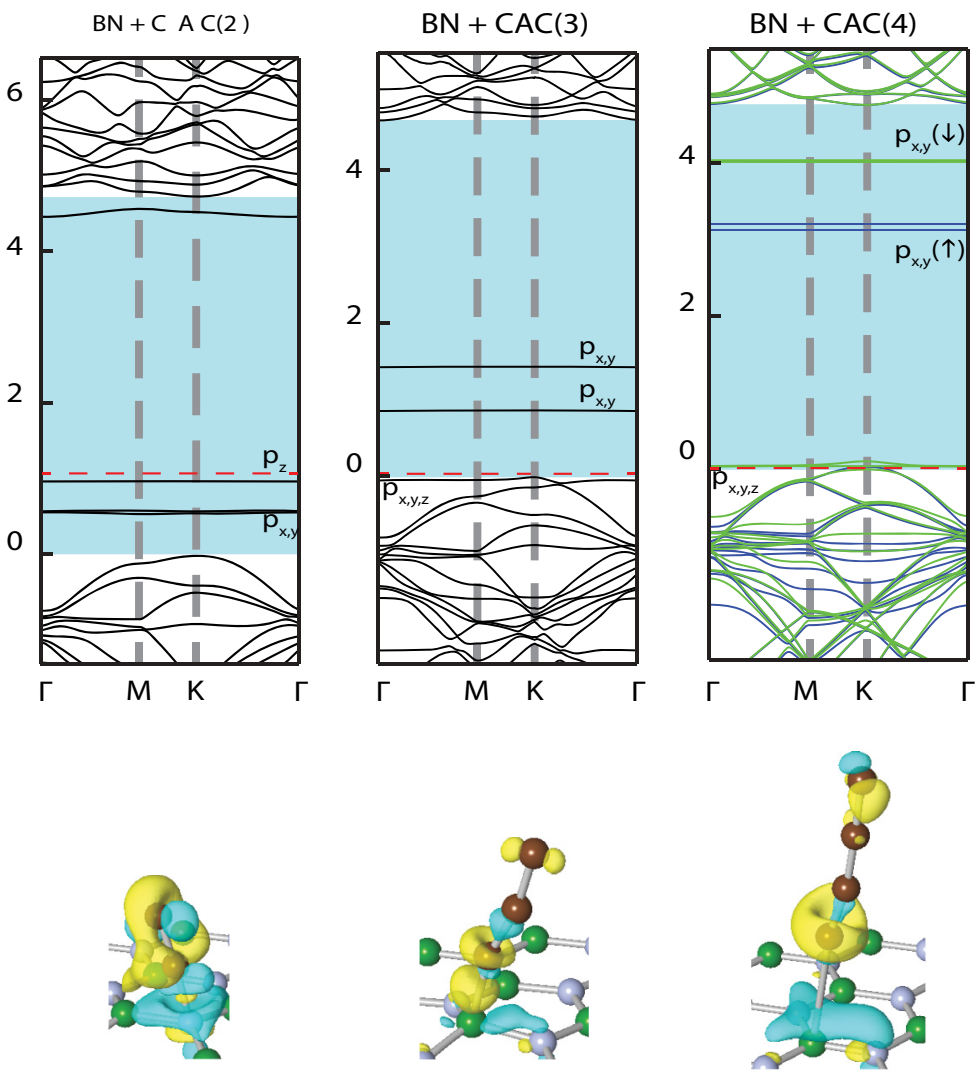

FIG. 6. (Color online) (a) Electronic-energy band structures of CACs grown on h-BN calculated for $n=1,2,3$ and 4 . In the magnetic cases, spin-up and -down bands are represented by blue and green lines, respectively. The bands below the red dashed-dotted lines are fully occupied. The localized impurity states appearing as flat bands in the band gap originate from the $p$ bands of the carbon atoms that are at the edges of chains. (b) Isosurfaces of the difference charge densities of chains where yellow and green regions designate charge accumulation and charge depletion, respectively. The isosurface values are taken as 0.01 electron $/ \AA^{3}$ for $C, C_{2}, C_{3}$ and as 0.005 electron $/ \AA^{3}$ for $C_{4}$.

for atomic-layer deposition techniques, it is conjectured that similar atomic-layer deposition or molecular-beam-epitaxy methods can be used for chain growth on BN layers at specific ambient conditions.

$\mathrm{BN}$ is in general not a chemically active material, however, with the inclusion of certain adatoms, its chemical activity can be improved. CACs grown on BN can be used for functionalization of h-BN layers which will enable the adsorption of certain adatoms such as hydrogen, lithium, and gold. This functionalization will especially be useful where the contact on an insulating surface such as $\mathrm{BN}$ is desired along with connection of electrodes. In order to increase the chemical activity of the inactive $\mathrm{BN}$ layer, we use the $\mathrm{CAC}(2)$ chain. In the previous sections, we showed that $\mathrm{CAC}(2)$ binds to $\mathrm{h}-\mathrm{BN}$ with a very high binding energy as compared to other CACs. Aside from being bonded strongly to h-BN, it also creates a chemically active site for the absorption of other adatoms.

Here, we test the adsorption of $\mathrm{Au}, \mathrm{Li}, \mathrm{Ti}, \mathrm{H}_{2}$, and $\mathrm{O}_{2}$ on $\mathrm{CAC}(2)$ and show that the chemical activity of $\mathrm{BN}$ can be enhanced through $\mathrm{CAC}(2)$ attached to it. Adatoms were placed in the vicinity of $\mathrm{CAC}(2)$ on $\mathrm{h}-\mathrm{BN}$ and fully self-consistent calculations were performed. Before introducing the adatoms, the $\mathrm{CAC}(2)$ was placed freely in its most favorable position as calculated in the previous section. It was seen that $\mathrm{Au}$ and $\mathrm{Li}$ atoms move towards the chain and are bound to the chemically active site of $\mathrm{CAC}(2)$ with binding energies of 1.77 and $1.53 \mathrm{eV}$, respectively. These are significantly higher than the binding energies of $\mathrm{Au}$ and $\mathrm{Li}$ on bare h-BN, which are calculated as 0.01 and $0.12 \mathrm{eV}$, respectively. This is an important result indicating that gold electrodes can easily be connected to semiconducting h-BN through CACs. A hydrogen molecule approaching to $\mathrm{CAC}(2)$ from the sides moves upwards and dissociates to form two $\mathrm{C}-\mathrm{H}$ bonds. The binding energy for $\mathrm{H}$ atoms is calculated as $3.62 \mathrm{eV}$. However, when $\mathrm{O}_{2}$ is introduced to the system instead of $\mathrm{H}_{2}$, it stays completely inactive and stays away from CAC(2). Finally, we consider the $\mathrm{Ti}$ adatom. Normally, $\mathrm{Ti}$ is bound to bare h-BN with an energy of $0.80 \mathrm{eV}$. However, when $\mathrm{h}-\mathrm{BN}$ is functionalized with $\mathrm{CAC}(2)$, although the Ti adatom initially is bound to the carbon atoms, it does not stay there but takes away these $\mathrm{CAC}(\mathrm{s})$ from the $\mathrm{BN}$ layer and forms a $\mathrm{TiC}_{2}$ structure which moves away from $\mathrm{BN}$. This final configuration is energetically more favorable than $\mathrm{Ti}$ binding to $\mathrm{h}-\mathrm{BN}$ by $5.9 \mathrm{eV}$. The final relaxed geometries of all these structures are presented in Fig. 7.

Pillared graphene structures and CACs passivated by graphene surfaces from both ends have also been subjects of recent interest of both theoretical and experimental studies. ${ }^{38}$ It was recently shown that CACs passivated by graphene flakes from both ends produce highly stable chain structures. ${ }^{39}$ Oxidized graphene pillars were also shown to be useful materials for storage applications. ${ }^{40}$ 

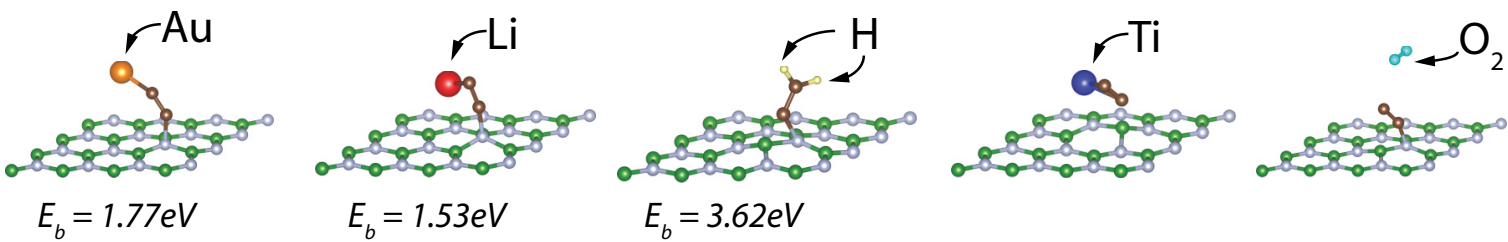

FIG. 7. (Color online) Functionalization of BN sheets through adsorption of carbon chains. For example, a CAC(2), which is strongly bound to h-BN, creates chemically active sites for $\mathrm{Au}, \mathrm{Li}$, and $\mathrm{H}$ atoms. $\mathrm{H}_{2}$ molecule approaching to $\mathrm{CAC}(2)$ from sides dissociated to form two $\mathrm{C}-\mathrm{H}$ bonds, whereas $\mathrm{O}_{2}$ remains totally inactive. Ti atom takes the carbon atoms with itself and forms $\mathrm{TiH}_{2}$.

Motivated by previous studies, we also calculated the bonding geometries of CACs between BN layers and showed that CACs can also be grown between two BN flakes as shown in Fig. 8. We have demonstrated this situation by calculating optimized bonding configurations and the spacing between two BN flakes when $\mathrm{CAC}(2)$ and $\mathrm{CAC}(3)$ are grown between them. $\mathrm{CAC}(2)$ is bonded to the nitrogen atom from one side and to the boron atom on the other side. On the other hand, CAC(3) is bonded to both of the flakes from the top of the boron atom. Once $\mathrm{CAC}(2)$ and $\mathrm{CAC}(3)$ are grown, the spacing between the flakes increases from $3.1 \AA$ to 4.34 and $5.82 \AA$, respectively. The formation energies, calculated by subtracting the energy of two BN planes and the energy of CAC from the energy of the final structure, are found as 2.0 and $0.32 \mathrm{eV}$ for $\mathrm{CAC}(2)$ and $\mathrm{CAC}(3)$, respectively.

\section{BN ATOMIC CHAINS GROWN ON GRAPHENE}

Having shown the stable carbon chain structures grown on $\mathrm{BN}$, we next investigate the growth of short $\mathrm{BN}$ chains on graphene to see if a similar self-assembly mechanism is also present for this reverted situation. Here, we adopt the representation where $\mathrm{BN}(n)+$ graphene indicates a $\mathrm{BN}$ chain consisting of $n$ atoms that is attached to graphene through the $\mathrm{N}$ atom. For example, $\mathrm{NB}(3)+$ graphene [or $\mathrm{NB}(3)$ only] stands for B-N-B chain attached to graphene through the $\mathrm{B}$ atom. Following the similar procedures described in Secs. III and IV, we calculate the most favorable binding sites, electronic properties, binding energies. We first perform MD simulations to see the self-assembly mechanisms of BN chains. To this end, we start by placing single $\mathrm{B}$ and $\mathrm{N}$ adatoms on the graphene supercell and run MD simulations at $500 \mathrm{~K}$ for $10 \mathrm{ps}$. Eventually, the two adatoms form a BN molecule perpendicularly attached to graphene. When a third adatom $\mathrm{N}$ is placed on the graphene at the close proximity of the existing $\mathrm{BN}$ molecule, MD simulations show that a chain comprising three atoms B-N-B forms. This procedure was repeated for
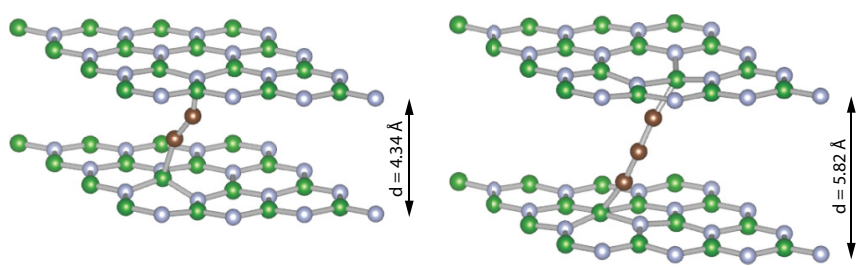

FIG. 8. (Color online) $\mathrm{CAC}(2)$ and $\mathrm{CAC}(3)$ grown between two $\mathrm{BN}$ flakes. The optimized spacing between the flakes increases from $3.1 \AA$ to $4.34 \AA$ and $5.82 \AA$ upon the formation of chains. different lengths, and it was observed that $\mathrm{B}$ and $\mathrm{N}$ atoms are indeed self-assembled on graphene honeycomb structure to form short BN atomic chains. Snapshots taken from these MD simulations are presented in Fig. 9.

The most favorable binding sites of short $\mathrm{BN}$ chains on graphene and their corresponding binding energies were calculated with self-consistent conjugate gradient calculations. It was found that the binding site of a BN chain on the graphene depends on the type of its closest atom to the graphene. A $\mathrm{NB}(n)$ chain is bound to the graphene layer at the bridge site, and a $\mathrm{BN}(n)$ is bound at the top site as shown in Fig. 10 . However, both single $\mathrm{B}$ and single $\mathrm{N}$ adatoms are bound at the bridge site. Contrary to the CAC case, no clear even/odd disparity was obtained in h-BN + graphene complexes owing to the ionic bonding between B-N atoms. Here, there is another effect related with the number of electrons in the chain. Chains having an odd number of atoms have an odd number of electrons, while even-numbered chains have an even number of electrons. The binding energies of these chains shown in Table II suggest that BN chains are bound with higher
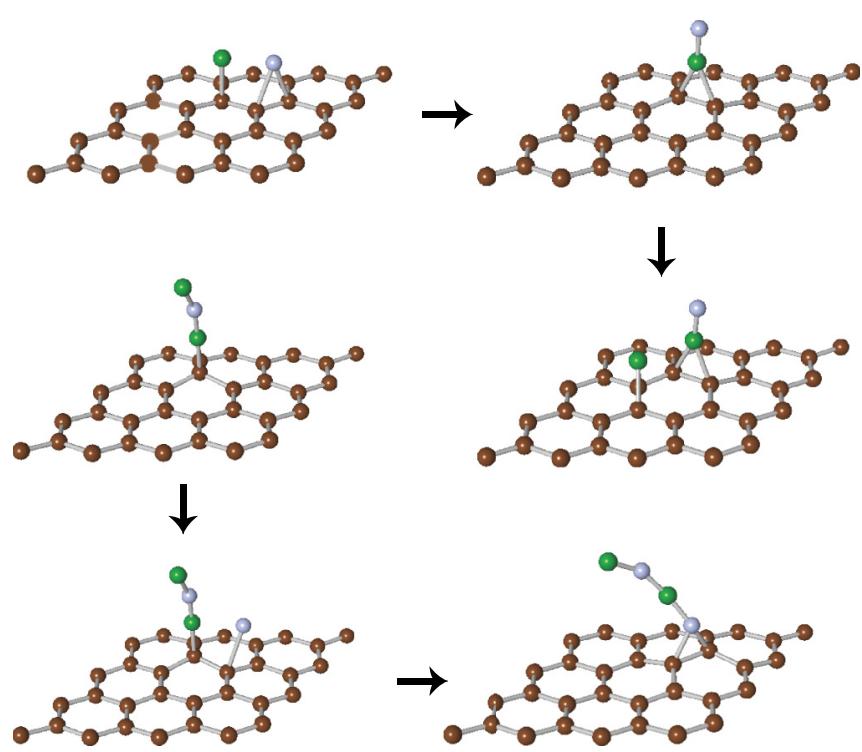

FIG. 9. (Color online) Growth dynamics of short NB chains on graphene. Once a new adatom is placed at a random point in the neighborhood of a NB chain having $n$ atoms, the structure rearranges itself to form a chain comprising $n+1$ atoms. The snapshots are taken from the quantum MD simulations performed at $500 \mathrm{~K}$ for 10 ps. Blue, green, and brown balls are, respectively, nitrogen, boron, and carbon atoms. 
(a)

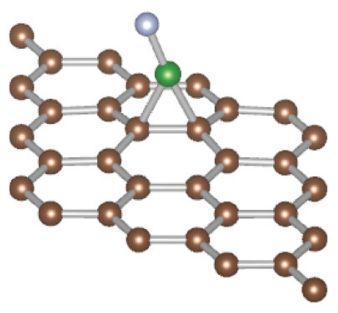

(b)

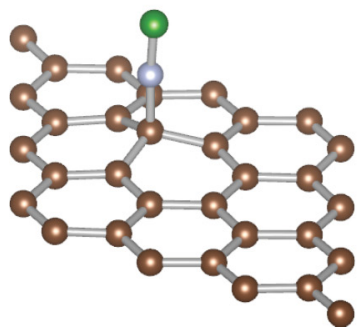

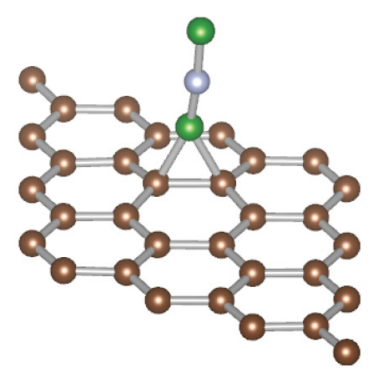

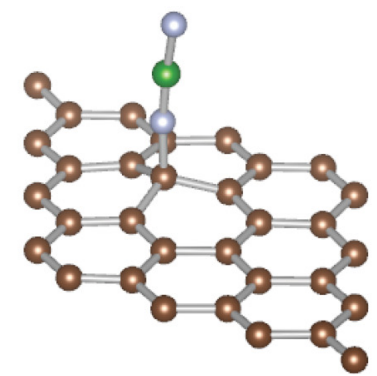

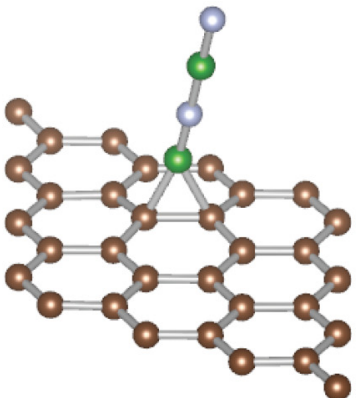

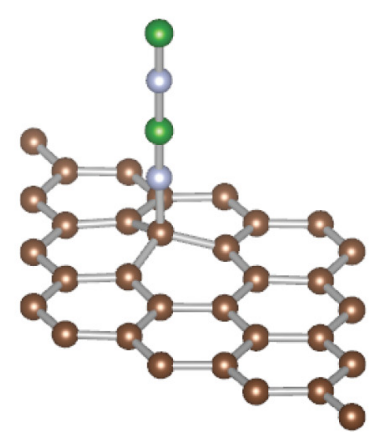

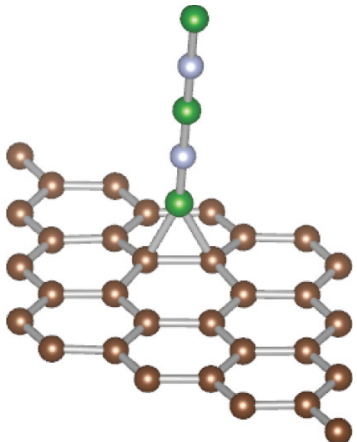

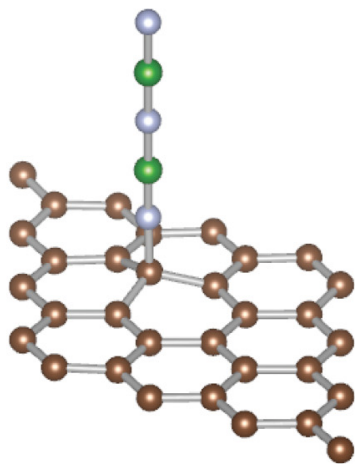

FIG. 10. (Color online) The most favorable binding configurations of short NB and BN chains grown on graphene. A NB(n) chain is attached to graphene at the bridge site since its nearest atom to graphene is $\mathrm{B}$, and $\mathrm{BN}(n)$ is bound at the top of the carbon atom since its nearest atom to graphene is $\mathrm{N}$. Energies are calculated using a $(4 \times 4)$ supercell and their stabilities are tested with MD simulations at $T=500 \mathrm{~K}$ for 10 ps. Note that the most favorable binding site is the bridge site for both single $\mathrm{N}$ and single $\mathrm{B}$ adatoms. Blue, green, and brown balls are, respectively, nitrogen, boron, and carbon atoms.

energies to the graphene as compared to the binding energies of CACs on BN, especially for longer chains. The binding energy of a boron-ending chain (i.e., NB) is always higher than a nitrogen-ending chain (i.e., $\mathrm{BN}$ ).

The even/odd disparity observed in the magnetic properties of $\mathrm{CAC}(n)+\mathrm{h}-\mathrm{BN}$ is also not seen for $\mathrm{BN}$ chains grown on

TABLE II. Most favorable binding sites, binding energies $\left(E_{b}\right)$, and the heights $(h)$ of $\mathrm{B}, \mathrm{N}$ adatoms and $\mathrm{BN}$ or $\mathrm{NB}$ atomic chains from the graphene plane. Here, for example, $\mathrm{BN}(3)$ indicates the $\mathrm{BN}$ chain consisting of three atoms (namely, N-B-N) grown on graphene with $\mathrm{N}$ being attached to graphene.

\begin{tabular}{llcr}
\hline \hline Structure & Binding site & $E_{b}(\mathrm{eV})$ & $h(\AA)$ \\
\hline B-adatom & Bridge & 1.24 & 1.45 \\
N-adatom & Bridge & 1.05 & 1.25 \\
$\mathrm{NB}(2)$ & Bridge & 2.58 & 1.46 \\
$\mathrm{BN}(2)$ & Top & 2.42 & 1.32 \\
$\mathrm{NB}(3)$ & Bridge & 1.18 & 1.52 \\
$\mathrm{BN}(3)$ & Top & 0.90 & 1.31 \\
$\mathrm{NB}(4)$ & Bridge & 0.82 & 1.58 \\
$\mathrm{BN}(4)$ & Top & 0.46 & 1.39 \\
$\mathrm{NB}(5)$ & Bridge & 0.73 & 1.63 \\
$\mathrm{BN}(5)$ & Top & 0.21 & 1.48 \\
$\mathrm{NB}(6)$ & Bridge & 0.51 & 1.67 \\
$\mathrm{BN}(6)$ & Top & 0.15 & 1.57 \\
\hline \hline
\end{tabular}

graphene. The single $\mathrm{B}$ adatom as well as single $\mathrm{N}$ adatom adsorbed on graphene have magnetic ground states. However, while $\mathrm{BN}(2)$ and $\mathrm{NB}(2)$ chains on graphene are nonmagnetic, $\mathrm{BN}(3)$ and $\mathrm{NB}(3)$ on graphene have magnetic ground states. This order is disturbed by the chain consisting of four atoms; while $\mathrm{BN}(4)+$ graphene is nonmagnetic, $\mathrm{NB}(4)+$ graphene has magnetic ground state.

The electronic structures calculated for a single adsorbate, namely, single boron and nitrogen adatoms, and single $\mathrm{NB}(2)$ and $\mathrm{BN}(2)$ molecules, perpendicularly attached to a $(4 \times 4)$ supercell of graphene, are shown in Fig. 11. Owing to their odd number of electrons, single $\mathrm{N}$ and $\mathrm{B}$ adatoms shift the Fermi level up and down relative to the Dirac point of graphene. This is an important feature to be used in electronic applications of single-layer graphene. For $\mathrm{NB}(2)+$ graphene, the bands crossing at the Fermi level attribute semimetallic character. On the other hand, $\mathrm{BN}(2)+$ graphene is a semiconductor with a narrow indirect band gap. This situation shows the dramatic effect of the type of chain atom which is attached to graphene. In addition, the supercell size, adsorbates-adsorbate coupling, as well as the symmetry of the adsorbate + supercell system are crucial for the resulting electronic structure for small supercells. ${ }^{41,42}$ For example, the semiconductor presented in Fig. 11(d) becomes metallic when the $\mathrm{BN}+$ graphene system is treated in a $(6 \times 6)$ supercell. In the case of a very large supercell having single adsorbate attached, the band crossing is recovered and the states of adsorbate appear as localized (resonance) impurity states. 
(a) B-Graphene

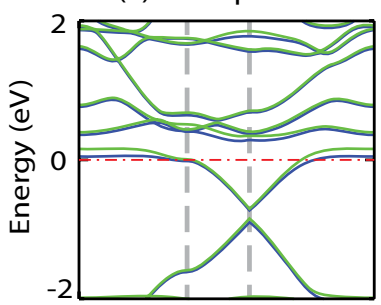

(c)NB-Graphene

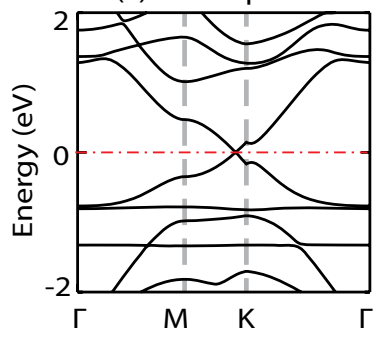

(b) N-Graphene

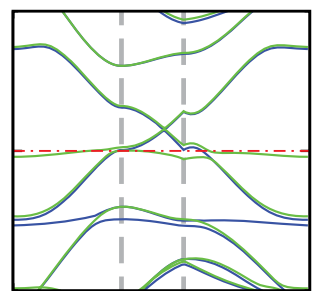

(d)BN-Graphene

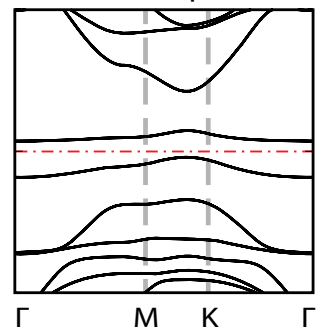

FIG. 11. (Color online) Electronic-energy structures: (a) Single $\mathrm{B}$ adatom adsorbed on graphene. (b) Single N adatom adsorbed on graphene. (c) NB chain consisting of two atoms grown on graphene with $\mathrm{B}$ atom being closest to graphene. (d) BN chain of two atoms grown on graphene with $\mathrm{N}$ being the closest adatom to graphene. The zero of energy is set to the Fermi energy, shown by red dashed-dotted line. In the magnetic cases, spin-up and -down bands are represented by blue (dark) and green (light) lines, respectively.

\section{CONCLUSION}

In conclusion, using first-principles calculations within the density-functional theory, we revealed the growth mechanism of carbon and $\mathrm{BN}$ atomic chains on single-layer honeycomb structures, namely, hexagonal $\mathrm{BN}$ (or h-BN) and graphene, respectively. We found that with the inclusion of each new carbon atom, the existing atomic chain consisting of $n$ carbon atoms leaves its previous position to join with the new carbon atom to form a chain of $n+1$ carbon atoms. Similar but more complex growth mechanisms were also found for BN chains grown on graphene layers depending on the type of chain atom ( $\mathrm{B}$ or $\mathrm{N}$ ) which is attached to graphene. These growth processes were simulated by ab initio molecular dynamics calculations at various temperatures, and the resulting chain structures were shown to be stable even though they are free to bend and slightly tilt. Nevertheless, these simulations are performed at high temperatures $(1000 \mathrm{~K})$ and the chains did not detach from the substrate plane for $10 \mathrm{ps}$, which is adequately long for ab initio molecular dynamics simulations. Therefore, these simulations present reliable stability results, especially at room temperature. The growth of atomic chains on the single-layer honeycomb structures heralds a self-assembly process, which may have fundamental and technological implications.

The grown chains by themselves exhibit interesting physical and chemical properties depending on the number of atoms forming the chain and the type of chain atom attaching to the substrate. In particular, the physical properties of even-numbered and odd-numbered carbon chains can behave differently leading to interesting even-odd disparity. We also showed that atomic chains grown on single-layer substrates attribute useful functionalities to bare h-BN and graphene. These properties are dependent on the number of carbon atoms in the chains. Apart from creating localized electronic states in the band gap and local magnetic moments, it is demonstrated that carbon chains can also be used for increasing the interlayer spacing between BN flakes, where specific molecules can be stored. Atomic chains grown on graphene and h-BN create chemically active sites on BN surface for atoms such as $\mathrm{Au}, \mathrm{Li}$, and $\mathrm{H}_{2}$, which provides the connection of these materials. Additionally, specific atoms forming strong bonds with atomic chains modify the electronic structure in the band gap and hence change the conductivity for possible sensor applications. Similarly, modifications of the electronic properties of graphene depending on the grown $\mathrm{B}$ or $\mathrm{N}$ adatoms and $\mathrm{BN}$ or NB chains are also worth emphasizing. Moving the Fermi level of graphene up or down by adsorbing $\mathrm{N}$ or $\mathrm{B}$ atoms, respectively, can be a worthwhile feature in the electronic applications related with graphene. The self-assembly in terms of atomic chains grown on semimetallic graphene and semiconducting h-BN and interesting functionalities achieved thereof can bring up new perspectives for further research on single-layer honeycomb structures.

\section{ACKNOWLEDGMENTS}

Part of the computational resources have been provided by TUBITAK ULAKBIM, High Performance and Grid Computing Center (TR-Grid e-Infrastructure) and UYBHM at Istanbul Technical University through Grant No. 2-024-2007. This work was supported partially by the Academy of Sciences of Turkey (TUBA). *ciraci@fen.bilkent.edu.tr

${ }^{1}$ K. S. Novoselov, A. K. Geim, S. V. Morozov, D. Jiang, Y. Zhang, S. V. Dubonos, I. V. Grigorieva, and A. A. Firsov, Science 306, 666 (2004).

${ }^{2}$ A. K. Geim and K. S. Novoselov, Nat. Mater. 6, 183 (2007).

${ }^{3}$ W. Paszkowicz, J. B. Pelka, M. Knapp, T. Szyszko, and S. Podsiadlo, Appl. Phys. A 75, 431 (2002).

${ }^{4}$ Z. Liu, L. Song, S. Zhao, J. Huang, L. Ma, J. Zhang, J. Lou, and P. M. Ajayan, Nano Lett. 11, 2032 (2011).

${ }^{5}$ K. Watanabe, T. Taniguchi, and H. Kanda, Nat. Mater. 3, 404 (2004).
${ }^{6}$ D. Pacile, J. C. Meyer, C. O. Girit, and A. Zettl, Appl. Phys. Lett. 92, 133107 (2008).

${ }^{7}$ N. G. Chopra, R. J. Luyken, K. Cherrey, V. H. Crespi, M. L. Cohen,

S. G. Louie, and A. Zettl, Science 269, 966 (1995).

${ }^{8}$ Y. J. Chen, H. Z. Zhang, and Y. Chen, Nanotechnology 17, 786 (2006).

${ }^{9}$ C. R. Dean, A. F. Young, I. Meric, C. Lee, L. Wang, S. Sorgenfrei, K. Watanabe, T. Taniguchi, P. Kim, K. L. Shepard, and J. Hone, Nat. Nanotechnol. 5, 722 (2010).

${ }^{10}$ C. Ataca and S. Ciraci, Phys. Rev. B 82, 165402 (2010). 
${ }^{11}$ A. Abdurahman, A. Skukla, and G. Seifert, Phys. Rev. B 66, 155423 (2002).

${ }^{12}$ S. Tongay, R. T. Senger, S. Dag, and S. Ciraci, Phys. Rev. Lett. 93, 136404 (2004).

${ }^{13}$ S. Tongay, S. Dag, E. Durgun, R. T. Senger, and S. Ciraci, J. Phys.: Condens. Matter 17, 3823 (2005).

${ }^{14}$ R. T. Senger, S. Tongay, S. Dag, E. Durgun, and S. Ciraci, Phys. Rev. B 71, 235406 (2005).

${ }^{15}$ R. T. Senger, S. Tongay, E. Durgun, and S. Ciraci, Phys. Rev. B 72, 075419 (2005).

${ }^{16}$ S. Dag, S. Tongay, T. Yildirim, E. Durgun, R. T. Senger, C. Y. Fong, and S. Ciraci, Phys. Rev. B 72, 155444 (2005).

${ }^{17}$ E. Durgun, R. T. Senger, H. Sevincli, H. Mehrez, and S. Ciraci, Phys. Rev. B 74, 235413 (2006).

${ }^{18}$ E. Durgun, R. T. Senger, H. Mehrez, S. Dag, and S. Ciraci, Europhys. Lett. 73, 642 (2006).

${ }^{19}$ S. Cahangirov, M. Topsakal, and S. Ciraci, Phys. Rev. B 82, 195444 (2010).

${ }^{20}$ M. Topsakal and S. Ciraci, Phys. Rev. B 81, 024107 (2010).

${ }^{21}$ S. Eisler, A. D. Slepkov, E. Elliott, T. Luu, R. McDonald, F. A. Hegmann, and R. R. Tykwinski, J. Am. Chem. Soc. 127, 2666 (2005).

${ }^{22}$ C. Jin, H. Lan, L. Peng, K. Suenaga, and S. Iijima, Phys. Rev. Lett. 102, 205501 (2009).

${ }^{23}$ W. A. Chalifoux, and R. R. Tykwinski, Nat. Chem. 2, 967 (2010).

${ }^{24}$ C. Ataca and S. Ciraci, Phys. Rev. B 83, 235417 (2011).

${ }^{25}$ J. C. Meyer, C. O. Girit, M. F. Crommie, and A. Zettl, Nature (London) 454, 319 (2008).

${ }^{26}$ W. Kohn and L. J. Sham, Phys. Rev. 140, A1133 (1965); P. Hohenberg and W. Kohn, ibid. 136, B864 (1964).

${ }^{27}$ M. C. Payne, M. P. Teter, D. C. Allen, T. A. Arias, and J. D. Joannopoulos, Rev. Mod. Phys. 64, 1045 (1992).
${ }^{28}$ P. E. Blochl, Phys. Rev. B 50, 17953 (1994).

${ }^{29}$ G. Kresse and D. Joubert, Phys. Rev. B 59, 1758 (1999).

${ }^{30}$ J. P. Perdew, J. A. Chevary, S. H. Vosko, K. A. Jackson, M. R. Pederson, D. J. Singh, and C. Fiolhais, Phys. Rev. B 46, 6671 (1992).

${ }^{31}$ S. Grimme, J. Comput. Chem 27, 1787 (2006).

${ }^{32}$ G. Kresse and J. Hafner, Phys. Rev. B 47, 558 (1993); G. Kresse and J. Furthmuller, ibid. 54, 11169 (1996).

${ }^{33}$ H. J. Monkhorst and J. D. Pack, Phys. Rev. B 13, 5188 (1976).

${ }^{34}$ In Ref. 10, the energy barrier for the migration of single carbon atom on h-BN was calculated to be $\sim 0.3 \mathrm{eV}$. In those calculations, the van der Waals correction was not included. For the same reasons, the present calculations with van der Waals correction predicts the binding energy as $0.95 \mathrm{eV}$ relative to the energy of the most favorable site, which is $\sim 0.25 \mathrm{eV}$ higher than that calculated without van der Waals correction.

${ }^{35}$ X. Fan, L. Liu, J. Lin, Z. Shen, and J. Kuo, ACS Nano 3, 3788 (2009).

${ }^{36}$ V. O. Özçelik, S. Cahangirov, and S. Ciraci, Phys. Rev. B 85, 235456 (2012).

${ }^{37}$ S. Haghighatpanah, A. Börjesson, H. Amara, C. Bichara, and K. Bolton, Phys. Rev. B 85, 205448 (2012).

${ }^{38}$ G. K. Dimitrakakis, E. Tylianakis, and G. E. Froudakis, Nano Lett. 8, 3166 (2008).

${ }^{39}$ L. Ravagnan, N. Manini, E. Cinquanta, G. Onida, D. Sangalli, C. Motta, M. Devetta, A. Bordoni, P. Piseri, and P. Milani, Phys. Rev. Lett. 102, 245502 (2009).

${ }^{40}$ J. W. Burress, S. Gadipelli, J. Ford, J. M. Simmons, W. Zhou, and T. Yildirim, Angew. Chem. Int. Ed. 49, 8902 (2010).

${ }^{41}$ H. Sahin and S. Ciraci, Phys. Rev. B 84, 035452 (2011).

${ }^{42} \mathrm{Ph}$. Lambin, H. Amara, F. Ducastelle, and L. Henrard, Phys. Rev. B 86, 045448 (2012). 